\title{
Molecular Weight Distribution and Intrinsic Viscosity of Sonicated and Successively Fractionated Double-Stranded Deoxyribonucleic Acid and Polyribonucleotides ${ }^{\dagger}$
}

\author{
Masato Tanigawa, Nobuaki Mukaiyama, Satoshi Shimokubo, \\ Kengo Wakabayashi, Yoshimasa Fujita, Kiyohiro Fukudome, \\ and Kiwamu YAMAOKA* \\ Department of Materials Science, Faculty of Science, Hiroshima University, \\ 1-3-1 Kagamiyama, Higashi-Hiroshima 724, Japan
}

(Received August 6, 1993)

\begin{abstract}
The ultrasonic scission was studied for deoxyribonucleic acid (DNA) and five polyribonucleotide duplexes; three antiparallel-stranded helices $(\operatorname{Poly}(G) \cdot \operatorname{Poly}(C)$, Poly(A) . $\operatorname{Poly}(\mathrm{U}), \operatorname{Poly}(\mathrm{I}) \cdot \operatorname{Poly}(\mathrm{C}))$ and two parallel-stranded helices $\left(\operatorname{Poly}(\mathrm{C}) \cdot \operatorname{Poly}\left(\mathrm{C}^{+}\right)\right.$and $\operatorname{Poly}(\mathrm{A}) \cdot$ Poly $\left(\mathrm{A}^{+}\right)$). The sonication was carried out in $0.1-0.2 \mathrm{M} \mathrm{NaCl}$ solutions at $0^{\circ} \mathrm{C}$ and 200 watts for totally 20 minutes under helium gas atmosphere. The sonicated sample was fractionated by successive precipitation with acetone as the precipitant. For each duplex, several fractions were obtained in large quantities. The weight-average molecular weight $M_{w}$, the degree of polydispersity $M_{w} / M_{n}$, and the molecular weight distribution were determined for unsonicated, sonicated, and fractionated samples by the gel-permeation-chromatography/low-angle-laser-light-scattering method. The $M_{w}$ values were reduced to a limit of $10^{5}$ and the $M_{w} / M_{n}$ values were narrowed to a 2-1.5 range by sonication. These values were further refined by fractionation. The molecular weight distribution profiles of well-defined fractions could be fitted by the logarithmic-normal function. The relationship between intrinsic viscosity and $M_{w}$ was evaluated for each duplex. The result showed that the rigidity or flexibility of polymer chain depends on the molecular weight of fractionated samples and the kind of duplex examined.
\end{abstract}

KEY WORDS Sonicated DNA and Polynucleotides / Molecular Weights / Molecular Weight Distribution / Intrinsic Viscosity / Precipitational Fractionation / GPC/LALLS /

It is well-known that the hydrodynamic and electric properties of the double-stranded helices of deoxyribonucleic acid (DNA) and ribonucleic acid (RNA) are sensitive to the chain length or molecular weight. ${ }^{1-5}$ In order to study the dependence of these physical quantities on the chain length, a large quantity of respective polymer is needed. In addition, the sample should be fractionated in such a way that the molecular weight and the weight distribution of each fraction are well-defined. The technique of sonication has been employed in the past to prepare fairly large batches of DNA samples, each of which was irradiated for a different period of time and at a varied power level to reduce molecular weights. ${ }^{2,6,7}$ Recently, a preparative technique was developed for narrowing the molecular weight and weight distribution of a sonicated DNA sample by successive precipitational fractionation with

* To whom correspondence should be addressed.

† This paper is Part V of "Ultrasonic Scission of Deoxyribonucleic Acid in Aqueous Solution." Part IV is ref 11. Part of this work was presented at the 41st Annual Meeting of the Polymer Society, Japan, held at Yokohama, May 26-29, 1992. See Polym. Prepr. Jpn., 41, 1090 (1992). 
acetone. $^{8,9}$ The effect of chain length on the thermal profile, together with the hydrodynamic and spectroscopic properties, was determined for purified DNA fractions. ${ }^{6,7,10-12}$

Some questions remain unresolved on how the sonication process affects the molecular weight and the distribution, and also on how the new precipitation technique improves these properties, mostly because of the difficulty of molecular weight determinations. The intrinsic viscosity $[\eta]$ of a DNA solution is convenient for estimating the weight-average molecular weight $M_{w}$. Eigner and Doty reported an expression between $[\eta]$ and $M_{w}$ for a number of DNA's from various sources. ${ }^{13}$ Their relationship often leads to an error, since the intrinsic viscosity is affected by the molecular weight distribution even for a given value of $M_{w}$. In the present series of studies, for example, a slightly different relationship was presented for DNA fractions with the lower molecular weights (hence, the less chain flexibility.) that were prepared from calf thymus DNA by sonication and precipitational fractionation. ${ }^{10-12}$

The objectives of this work are (1) to establish that the molecular weights of both the antiparallel heteroduplexes in the A-form conformation and the parallel homoduplexes in slightly acidic region can be reduced in a similar manner as DNA, and (2) to present the [ $\eta]$ vs. $M_{w}$ relationship with the fractionated samples. For this purpose, the ultrasonic scission work was extended to five high molecular weight double-stranded polyribonucleotides and to calf thymus DNA as the reference. Both intact and sonicated samples were subjected to a newly acquired method, i.e., the gel-permeation-chromatography/lowangle-laser-light-scattering (abbreviated as GPC/LALLS), for the molecular weight, the weight distribution, and the polydispersity in terms of weight-average to number-average molecular weights $M_{w} / M_{n}$.

\section{EXPERIMENTAL}

\section{Materials and Ultrasonic Scission}

A calf thymus DNA sample was purchased from Worthington Biochemical Corp. (U.S.A.), lot no. 38C137. It was gently dissolved in $0.2 \mathrm{M} \mathrm{NaCl}$ solution. ${ }^{8,9}$ The following polyribonucleotide samples were either purchased from or kindly supplied by Yamasa Shoyu Co., Ltd. (Choshi, Chiba, Japan): poly(inosinic acid) $\cdot$ poly(cytidylic acid) (abbreviated as Poly $(\mathrm{I}) \cdot \operatorname{Poly}(\mathrm{C})$ ) was prepared from Poly(I), lot no. 501064, and Poly(C), lot no. 301270, by mixing in $0.2 \mathrm{M} \mathrm{NaCl}$ solution ${ }^{13-15}$; poly(adenylic acid) $\cdot$ poly(uridylic acid) (abbreviated as Poly(A) Poly(U)), lot no. 2-7, was dissolved in $0.1 \mathrm{M}$ $\mathrm{NaCl}^{16-19}$; a singly charged poly(cytidylic acid) - poly (cytidylic acid) (abbreviated as Poly$(\mathrm{C}) \cdot \operatorname{Poly}\left(\mathrm{C}^{+}\right)$) was prepared by dissolving Poly $(\mathrm{C})$, lot no. 301430 , in $0.1 \mathrm{M} \mathrm{NaCl}$ solution maintained at a $\mathrm{pH}$ of 4.5 with an acetic acid buffer at an ionic strength of $0.05^{20-23}$; a singly charged poly(adenylic acid) poly(adenylic acid) (abbreviated as $\operatorname{Poly}(\mathrm{A}) \cdot \operatorname{Poly}\left(\mathrm{A}^{+}\right)$) was prepared by dissolving Poly(A), lot no. S-101, in an acetic acid buffer maintained at a $\mathrm{pH}$ of 5.5 and at an ionic strength of $0.0871 . .^{24-26}$ The antiparallel double-stranded poly(guanylic acid) - poly(cytidylic acid) (abbreviated as Poly(G) $\cdot \operatorname{Poly}(C))$ was prepared by mixing Poly $(G)$, purchased from Sigma Chemical Co. (U.S.A.), lot no. 18F-4026, with Poly(C), kindly supplied by Yamasa Shoyu Co., Ltd., lot no. S-301, in $0.1 \mathrm{M} \mathrm{NaCl}$ solution maintained at a $\mathrm{pH}$ of 8 with a sodium phosphate buffer at an ionic strength of $0.05 . .^{27-29}$ It should be noted that the ionic strengths specified above are critical in some cases and that the references cited should be consulted with for actual sample preparations. Other chemicals were all of reagent grade.

Ultrasonic irradiation was carried out with Tomy sonicator, Model UR-200P (Tomy Seiko Co. (Tokyo)), at an output power level of 200 watts $(20 \mathrm{kHz})$ for totally 20 minutes 
(forty repetitions of a 30-second burst and a subsequent 5-minute bubbling interval) under the helium gas atmosphere. A polymer solution (usually $2.0 \mathrm{mg} \mathrm{ml}^{-1}$ but $1.5 \mathrm{mg} \mathrm{ml}^{-1}$ for $\left.\operatorname{Poly}(\mathrm{A}) \cdot \operatorname{Poly}\left(\mathrm{A}^{+}\right)\right)$was added in a three-way branched rosette vessel ( $c a .150 \mathrm{ml})$, in which the solution was vigorously circulated by jet flows for rapid temperature equilibration. The vessel was held at $0^{\circ} \mathrm{C}$ in an iced bath and the air was replaced by bubbling helium gas. Details of the sonication procedure have been described elsewhere. ${ }^{2,8,9}$ No damage in nucleic acid bases nor strand separation of duplex was detected by the UV absorption spectra of the polymer solution before and after the sonication.

All six sonicated sample solutions were fractionated to several fractions at $20^{\circ} \mathrm{C}$ by the successive precipitation method with acetone as the precipitant. ${ }^{8,9,12}$ Prior to viscosity and GPC/LALLS measurements, the fractions were dialyzed against appropriate $\mathrm{NaCl}$-added blank solutions at $4^{\circ} \mathrm{C}$ over 48 hours, with totally $4 \mathrm{~L}$ (several batches were changed), the first batch containing $0.1 \mathrm{M}$ ethylenediaminetetraacetate (disodium salt).

\section{Measurements}

$G P C / L A L L S$. The molecular weight and the distribution pattern were determined at $30^{\circ} \mathrm{C}$ on a Tosoh flow-type GPC/LALLS detecting system (Tosoh Co. (Tokyo)), which consists of two Tosoh TSK G-DNA-PW columns in tandem, a Tosoh LS-8000 light scattering photometer, and a Tosoh RI-8011 differential refractometer. A sample solution $(0.5 \mathrm{ml})$ at a concentration of $0.114-2.33 \mathrm{mg} \mathrm{ml}^{-1}$ was injected for a single run at a flow rate of $0.6 \mathrm{ml} \mathrm{min}^{-1}$. The eluting solution was filtered through a sintered stainless filter for the GPC column, and through a $0.45 \mu \mathrm{m}$ pore-size cellulose acetate membrane (Millipore Co., HAWP 01300) in front of the LALLS apparatus for complete removal of dusts. A bovine serum albumin sample (molecular weight $=66250$ ) from Miles Inc. Diagnostics

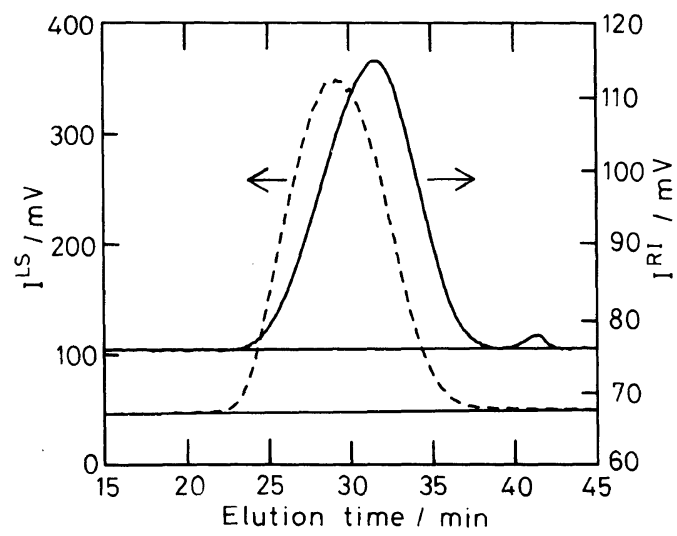

Figure 1. Chromatogram of a fractionated Poly(A). Poly(U) sample (F4) in $0.1 \mathrm{M} \mathrm{NaCl}$ through the GPC/ LALLS system. Conditions for detection: GPC column at $30^{\circ} \mathrm{C}$, refractive index at $35^{\circ} \mathrm{C}$ (right) and light scattering at room temperature (left).

Div., (U.S.A.), and a chicken erythrocyte nucleosomal DNA sample (molecular weight = 95700) were used as the molecular weight standards. Because of an aggregating tendency, the strands of $\operatorname{Poly}(\mathrm{C}) \cdot \operatorname{Poly}\left(\mathrm{C}^{+}\right)$were separated at a $\mathrm{pH}$ of 8 in a phosphate buffer adjusted at an ionic strength of 0.1 . The molecular weight was determined as the single strand and it was then multiplied by a factor of 2. $\operatorname{Poly}(\mathrm{A}) \cdot \operatorname{Poly}\left(\mathrm{A}^{+}\right)$was measured in a $0.05 \mathrm{M} \mathrm{NaCl}$ solution at a $\mathrm{pH}$ of 5.5 adjusted with acetate buffer at an ionic strength of 0.05 to avoid aggregation. The remaining polymer samples were all measured in the double-strand structure under the same conditions as described in Materials.

Figure 1 shows a typical GPC/LALLS chromatogram of a sonicated $\operatorname{Poly}(\mathrm{A}) \cdot \operatorname{Poly}(\mathrm{U})$ fraction taken on the present apparatus. The chromatogram was detected as the output signal intensities in voltage of the differential refractive indices, $I^{\mathrm{RI}}$, and of the scattered light, $I^{\mathrm{LS}}$, against the elution time. The small hump detected by refractive index is due to added salt in solvent.

\section{Analysis of GPC/LALLS Data}

Molecular Weights. The signal intensity of a 
refractive index $\Delta I^{\mathrm{RI}}$ and the signal intensity of a light scattering at a low angle $\Delta I^{\mathrm{LS}}$ are given for a dilute solution as

$$
\begin{gathered}
\Delta I^{\mathrm{RI}}=I_{\text {soln }}^{\mathrm{RI}}-I_{\text {solv }}^{\mathrm{RI}}=k_{1} c^{\prime} \\
\Delta I^{\mathrm{LS}}=I_{\text {soln }}^{\mathrm{LS}}-I_{\text {solv }}^{\mathrm{LS}}=k_{2} c^{\prime} M
\end{gathered}
$$

where $k_{1}$ and $k_{2}$ are constants, $c^{\prime}$ and $M$ are the mass concentration in mononucleotide unit and the molecular weight of a particular polymer molecule, respectively. From eq 1 and 2 , the following results:

$$
\Delta I^{\mathrm{LS}} / \Delta I^{\mathrm{RI}}=\left(k_{2} / k_{1}\right) M
$$

By eluting the standard solution, which contains a monodisperse sample of known molecular weight $M$ and concentration $c^{\prime}$, through the GPC/LALLS system, the instrumental constants $\left(k_{2} / k_{1}\right)$ can be evaluated. After determining the concentration of a sample whose molecular weight to be measured, the sample solution is now eluted through the GPC/LALLS system. From the observed intensities, the ratio, $\Delta I^{\mathrm{LS}} / \Delta I^{\mathrm{RI}}$, can be obtained, and finally $M$ can be calculated.

Molecular Weight Distribution. Being eluted through the GPC column, a sonicated and subsequently fractionated sample exhibits a characteristic elution pattern, with the highest molecular weight portion first entering the light scattering cell. From the refractive index and light scattering measurements of the sample, the molecular weight $M$ can be determined ( $c f$. eq 3). From the former measurement, the weight fraction $f_{w}(M)$ for the molecules with the molecular weight $M$ can be evaluated (cf. eq 1). In order to compare the molecular weight distribution over a wide range of $M$, another weight fraction $g_{w}(\log M)$ should be adopted, which is a function of $\log M$ and given as

$$
\begin{aligned}
f_{w}(M) \mathrm{d} M & =f_{w}(M)\left(\frac{\mathrm{d} M}{\mathrm{~d} \log M}\right) \mathrm{d} \log M \\
& \equiv g_{w}(\log M) \cdot \mathrm{d} \log M
\end{aligned}
$$

Hence, the molecular weight distribution can finally be calculated as the $g_{w} v s . \log M$ plot.

Viscosity. The intrinsic viscosity was measured at $25^{\circ} \mathrm{C}$ on a five-bulb Ubbelohde-type dilution viscometer for high molecular weight samples, which exhibit shear-rate dependence (DNA, Poly(A) · Poly(U), Poly(I) · Poly(C), and $\left.\operatorname{Poly}(\mathrm{A}) \cdot \operatorname{Poly}\left(\mathrm{A}^{+}\right)\right)$and on a single-bulb viscometer for other solutions. The observed reduced viscosities were extrapolated to zero shear-rate, whenever necessary, to evaluate the intrinsic viscosity. Both sample solution and solvent were filtered through a G-2 or G-3 glass filter to remove dusts. The solvent compositions were the same as those for GPC/LALLS measurements, except for $\operatorname{Poly}(\mathrm{C}) \cdot \operatorname{Poly}\left(\mathrm{C}^{+}\right)$, for which the buffer solution was the same as used for the duplex preparation.

\section{RESULTS AND DISCUSSION}

\section{Effect of Sonication and Fractionation on \\ Molecular Weight and Distribution}

Figures $2 \mathrm{a}-\mathrm{f}$ show the profiles of molecular weight distribution for six different polymer samples, each being fractionated by precipitation to several fractions as numbered from the first (F1) to the last, together with the sonicated but unfractionated (s) and the original and unsonicated (h) samples. The GPC/LALLS data are summarized in Table $\mathrm{I}$, where the weight-average molecular weight $M_{w}$, the ratios of weight-average to number-average molecular weights $M_{w} / M_{n}$ and those of z-average to weight-average molecular weights $M_{z} / M_{w}$.

$D N A$. In Figure $2 \mathrm{a}$, the s sample shows a slight tailing to the lower molecular weight side, while the F1 to F5 fractions show tailings to higher molecular weights. By sonication, the molecular weight is lowered to about one thirty-eighth (s) and further to about one hundredth by fractionation (F5). As the precipitational fractionation proceeds, the $M_{w} / M_{n}$ values become smaller, and hence the molecular weight distribution becomes nar- 
rower. The $M_{z} / M_{w}$ values of F1-F3 are higher than the value of s-sample, probably because of the coprecipitation of a small amount of very high molecular weight molecules.

$\operatorname{Poly}(G) \cdot \operatorname{Poly}(C)$. Poly $(\mathrm{G}) \cdot \operatorname{Poly}(\mathrm{C})$ forms an antiparallel double-stranded helix just as DNA. Since the molecular weight of the original Poly $(G)$ is relatively small, the duplex was degraded only slightly by sonication, as shown in Figure $2 \mathrm{~b}$ and also in Table I. This result is in conformity with the previous report for DNA that there is a practical lower limit in molecular weight degradation. ${ }^{8,9}$ Some $M_{w}$ values of acetone-precipitated fractions are indeed higher than the value of the starting sample ( $c f$. h-fraction and the others in Table I). Nevertheless, the sonication and fractionation technique is effective for preparing samples with various molecular weights in large quantities.

$\operatorname{Poly}(A) \cdot \operatorname{Poly}(U)$ and $\operatorname{Poly}(I) \cdot \operatorname{Poly}(C)$. These heteroduplexes form antiparallel doublestranded helices under the present conditions. Contrary to $\operatorname{Poly}(G) \cdot \operatorname{Poly}(C)$, the molecular weight of the sonicated Poly(A) $\operatorname{Poly}(\mathrm{U})$ was reduced to one third of the starting value, which is over a million (h-sample); the same is also true for $\operatorname{Poly}(\mathrm{I}) \cdot \operatorname{Poly}(\mathrm{C})$, as are shown in Figures $2 \mathrm{c}$ and $\mathrm{d}$ and also in Table I. Here again, the ultrasonic scission is quite effective for preparing samples with different chain lengths. The molecular weight distribution was also narrowed, some distribution profiles showing a tailing trend toward higher or lower molecular weight side. This irregularity is mostly due to the technical skill or experience of individuals who actually performed the precipitational fractionation protocol.

$\operatorname{Poly}(C) \cdot \operatorname{Poly}\left(C^{+}\right)$and $\operatorname{Poly}(A) \cdot \operatorname{Poly}\left(A^{+}\right)$. These homoduplexes are the parallel doublestranded helices in the acidic $\mathrm{pH}$ region. The molecular weight of the starting $\operatorname{Poly}(\mathrm{C})$. Poly $\left(\mathrm{C}^{+}\right)$was indeed reduced by half after sonication, and the fractionation with acetone seems quite successful, as judged from Figure $2 \mathrm{e}$ and Table $\mathrm{I}$. This result is remarkable in that the present procedure can produce samples with widely varying $M_{w}$ values regardless of the conformations of duplexes in solution. The molecular weight of the original Poly(A). Poly $\left(\mathrm{A}^{+}\right)$sample was too high to be determined by GPC/LALLS because of the GPC column presently in use; the sample easily form aggregates in acid solution. Figure $2 \mathrm{f}$ shows that each $\operatorname{Poly}(\mathrm{A}) \cdot \operatorname{Poly}\left(\mathrm{A}^{+}\right)$fraction contains higher molecular weight components, probably except for the smallest fraction (F4), as indicated with the tailing trend in the distribution profiles. On further reflection, it would be better if a high molecular weight single-stranded Poly(A) was first sonicated to prepare shorter fragments, and then the $\mathrm{pH}$ of the solution was lowered to 5.5 to form duplex fragments. This solution might then be subjected to re-sonication, in order to remove unpaired (or unmatched) ends from the duplex chains. The re-sonicated $\operatorname{Poly}(\mathrm{A}) \cdot \operatorname{Poly}\left(\mathrm{A}^{+}\right)$ solution could be fractionated to separate closely monodisperse fractions for studies of the solution properties.

Table I shows the recovery of the sonicated and fractionated samples for each polynucleotide duplex. The total recovery is generally satisfactory, ranging between 70 and $90 \%$ without appreciable loss of polymeric materials during manipulations. This result indicates that extremely low molecular weight materials are hardly produced by sonication, unless a prolonged irradiation time is adopted, and that the amount of a precipitated sample with a specified molecular weight may be controlled by adjusting the volume of added acetone. Finally, it should be noted that experience always counts most for executing the sonication-fractional precipitation procedure cited here.

\section{Comparison of Molecular Weight Distribution with Theoretical Distribution Function}

In the strict analysis of experimental results of a given polymer system, e.g., the hydrodynamic $^{10,30-32}$ and electro-optical ${ }^{1,3,33,34}$ 

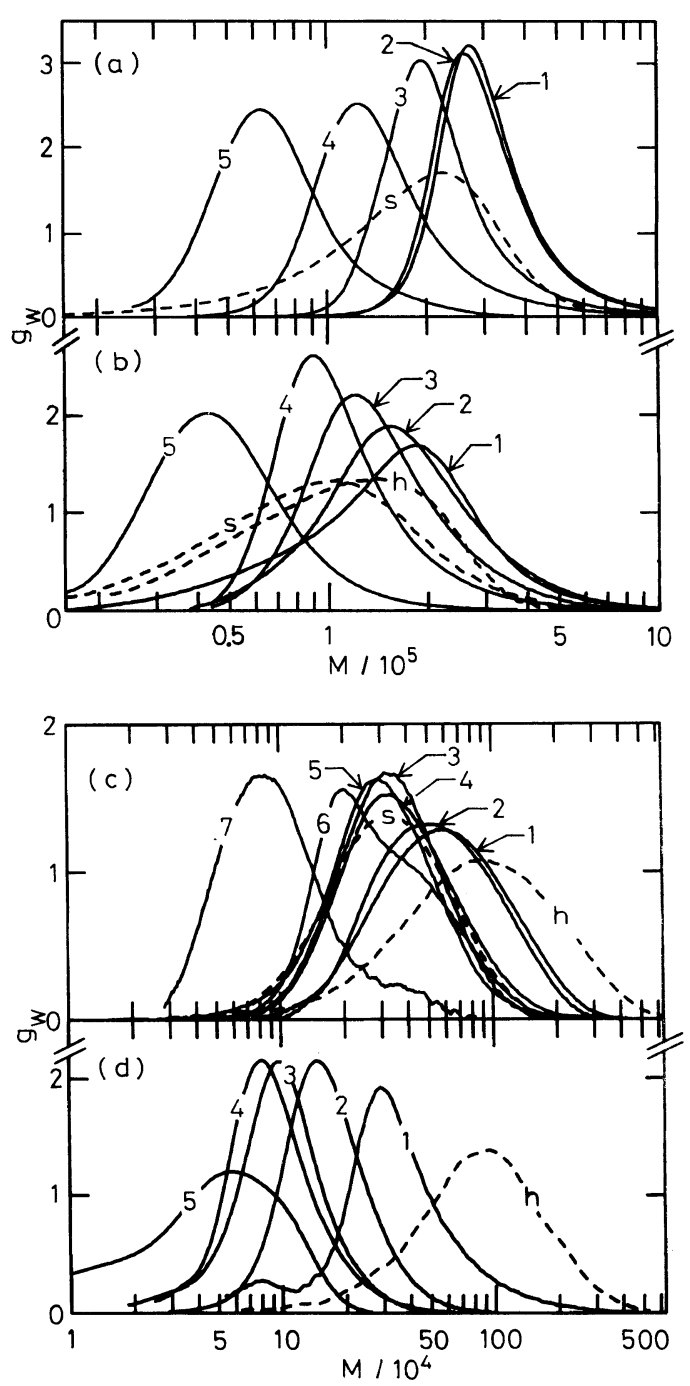

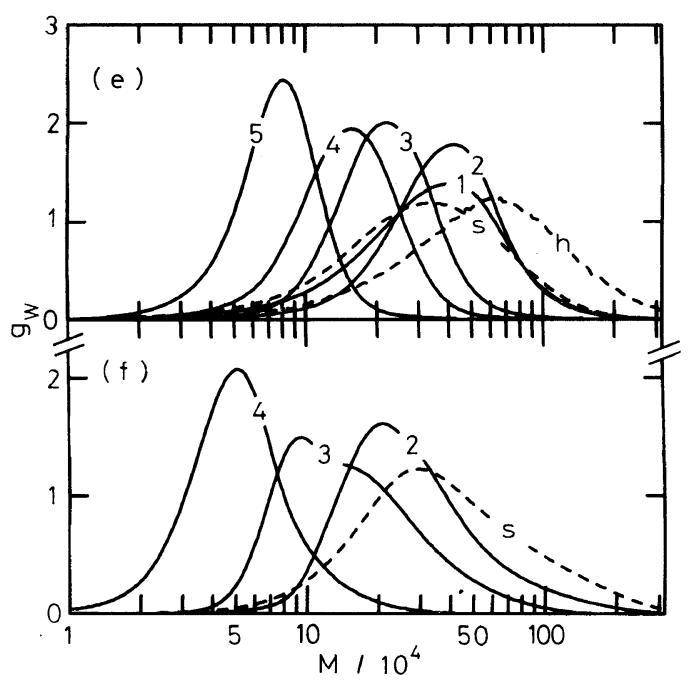

Figure 2. The GPC/LALLS-determined molecular weight distributions of the unsonicated (h), the sonicated but unfractionated (s) and the sonicated and fractionated (numbered from 1 to appropriate fraction number) doublestranded helix samples. The weight fraction $g_{w}$ was plotted against the molecular weight $M$ on a semilogarithmic scale. (a) DNA; (b) Poly(G) $\cdot \operatorname{Poly}(\mathrm{C})$; (c) $\operatorname{Poly}(\mathrm{A}) \cdot \operatorname{Poly}(\mathrm{U})$; (d) $\operatorname{Poly}(\mathrm{I}) \cdot \operatorname{Poly}(\mathrm{C})$; (e) Poly(C) $\operatorname{Poly}\left(\mathrm{C}^{+}\right)$; (f) Poly(A). $\operatorname{Poly}\left(\mathrm{A}^{+}\right)$.

Table I. Weight-average molecular weight, $M_{w}$, molecular weight distribution, $M_{w} / M_{n}$ and $M_{z} / M_{w}$, recovery, $Y$, added acetone, $W$, and intrinsic viscosity, $[\eta]$, of unsonicated (h), sonicated (s) and fractionated $(\mathrm{F} 1-\mathrm{F} 7)$ polynucleotide duplex samples

\begin{tabular}{|c|c|c|c|c|c|c|c|}
\hline \multirow{2}{*}{ Sample } & \multirow{2}{*}{ Fraction } & $M_{w}$ & \multirow{2}{*}{$M_{w} / M_{n}$} & \multirow{2}{*}{$M_{z} / M_{w}$} & $Y^{\mathrm{a}}$ & \multirow{2}{*}{$\frac{W^{\mathrm{b}}}{\%}$} & \multirow{2}{*}{$\frac{[\eta]}{10^{2} \mathrm{~cm}^{3} \mathrm{~g}^{-1}}$} \\
\hline & & $\overline{10^{4}}$ & & & $\%$ & & \\
\hline \multirow[t]{7}{*}{ DNA } & $\mathrm{h}$ & 780 & $-^{c}$ & $-^{c}$ & 100 & & 47.3 \\
\hline & s & 20.6 & 1.64 & 1.36 & & & 1.39 \\
\hline & $\mathrm{F} 1$ & 39.9 & 1.36 & 4.82 & 4.7 & 65.1 & 2.34 \\
\hline & $\mathrm{F} 2$ & 36.2 & 1.27 & 2.34 & 10.8 & 69.2 & 2.22 \\
\hline & F3 & 25.5 & 1.25 & 2.23 & 14.5 & 68.8 & 1.57 \\
\hline & F4 & 16.5 & 1.25 & 1.50 & 19.5 & 76.0 & 0.89 \\
\hline & F5 & 7.61 & 1.20 & 1.26 & $\begin{array}{c}6.4 \\
(55.9)\end{array}$ & 120.0 & 0.30 \\
\hline
\end{tabular}


GPC/LALLS of Sonicated Nucleic Acids

Table I. (Continued)

\begin{tabular}{|c|c|c|c|c|c|c|c|}
\hline \multirow{2}{*}{ Sample } & \multirow{2}{*}{ Fraction } & \multirow{2}{*}{$\frac{M_{w}}{10^{4}}$} & \multirow{2}{*}{$M_{w} / M_{n}$} & \multirow{2}{*}{$M_{z} / M_{w}$} & \multirow{2}{*}{$\frac{Y^{\mathrm{a}}}{\%}$} & \multirow{2}{*}{$\frac{W^{\mathrm{b}}}{\%}$} & \multirow{2}{*}{$\frac{[\eta]}{10^{2} \mathrm{~cm}^{3} \mathrm{~g}^{-1}}$} \\
\hline & & & & & & & \\
\hline \multirow[t]{7}{*}{$\operatorname{Poly}(\mathrm{G}) \cdot \operatorname{Poly}(\mathrm{C})$} & $\mathrm{h}$ & 12.9 & 1.51 & 1.42 & 100 & & 0.54 \\
\hline & s & 11.1 & 1.61 & 1.53 & & & 0.41 \\
\hline & $\mathrm{F} 1$ & 18.5 & 1.58 & 1.56 & 11.2 & 64.4 & 0.58 \\
\hline & $\mathrm{F} 2$ & 19.1 & 1.33 & 1.41 & 8.7 & 66.4 & 0.67 \\
\hline & F3 & 15.8 & 1.25 & 1.35 & 8.4 & 71.5 & 0.54 \\
\hline & $\mathrm{F} 4$ & 12.0 & 1.21 & 1.34 & 9.3 & 76.6 & 0.39 \\
\hline & F5 & 5.34 & 1.28 & 1.46 & $\begin{array}{c}13.4 \\
(51.0)\end{array}$ & 110 & 0.16 \\
\hline \multirow[t]{9}{*}{$\operatorname{Poly}(A) \cdot \operatorname{Poly}(\mathrm{U})$} & $\mathrm{h}$ & 113 & 2.17 & 1.67 & 100 & & 6.12 \\
\hline & s & 39.2 & 1.56 & 1.46 & & & 1.63 \\
\hline & $\mathrm{F} 1$ & 72.3 & 1.58 & 1.49 & 3.3 & 58.3 & 2.35 \\
\hline & $\mathrm{F} 2$ & 66.2 & 1.48 & 1.44 & 5.3 & 60.1 & 2.27 \\
\hline & F3 & 38.5 & 1.35 & 1.33 & 22.3 & 73.2 & 2.07 \\
\hline & F4 & 43.4 & 1.43 & 1.45 & 4.9 & 63.9 & 1.72 \\
\hline & F5 & 36.3 & 1.50 & 1.48 & 4.8 & 67.5 & 1.66 \\
\hline & F6 & 36.5 & 1.47 & 1.51 & 6.9 & 72.0 & 1.39 \\
\hline & F7 & 12.1 & 1.46 & 1.67 & $\begin{array}{c}2.8 \\
(50.3)\end{array}$ & 100 & 0.49 \\
\hline \multirow[t]{8}{*}{$\operatorname{Poly}(\mathrm{I}) \cdot \operatorname{Poly}(\mathrm{C})$} & $\mathrm{h}$ & 103 & 1.73 & 1.53 & 100 & & 6.22 \\
\hline & $\mathrm{s}$ & $\ldots^{d}$ & $-^{\mathrm{d}}$ & $-^{d}$ & & & 0.91 \\
\hline & $\mathrm{F} 1$ & 42.3 & 1.63 & 1.86 & 6.0 & 66.8 & 1.21 \\
\hline & $\mathrm{F} 2$ & 29.6 & 1.38 & 1.46 & 51.4 & 70.0 & 1.18 \\
\hline & F3 & 18.0 & 1.25 & 1.30 & 31.5 & 74.5 & 0.75 \\
\hline & $\mathrm{F} 4$ & 10.9 & 1.31 & 1.30 & 5.8 & 120 & 0.30 \\
\hline & F5 & 10.2 & 1.28 & 1.38 & 9.2 & 86.5 & 0.29 \\
\hline & F6 & 5.71 & 1.91 & 1.49 & $\begin{array}{c}2.4 \\
(106.3)\end{array}$ & 126 & 0.09 \\
\hline \multirow[t]{7}{*}{$\operatorname{Poly}(C) \cdot \operatorname{Poly}\left(\mathrm{C}^{+}\right)$} & $\mathrm{h}$ & 72.6 & 2.04 & 1.63 & 100 & & 2.03 \\
\hline & $\mathrm{s}$ & 40.3 & 2.01 & 1.66 & & & 1.21 \\
\hline & $\mathrm{F} 1$ & 41.5 & 1.85 & 1.54 & 22.6 & 73.8 & 2.17 \\
\hline & $\mathrm{F} 2$ & 44.7 & 1.56 & 1.42 & 19.9 & 77.2 & 1.31 \\
\hline & F3 & 24.5 & 1.33 & 1.38 & 4.3 & 86.5 & 0.79 \\
\hline & F4 & 16.9 & 1.39 & 1.41 & 12.3 & 81.7 & 0.51 \\
\hline & F5 & 8.36 & 1.29 & 1.29 & $\begin{array}{c}5.3 \\
(64.4)\end{array}$ & 100 & 0.29 \\
\hline \multirow[t]{6}{*}{$\operatorname{Poly}(\mathrm{A}) \cdot \operatorname{Poly}\left(\mathrm{A}^{+}\right)$} & $\mathrm{h}$ & - $^{\mathrm{e}}$ & $-^{e}$ & $-^{e}$ & 100 & & 83.0 \\
\hline & $\mathrm{s}$ & 51.0 & 1.93 & 1.98 & & & 5.60 \\
\hline & $\mathrm{F} 1$ & 244 & $-^{c}$ & $-^{c}$ & 17.4 & 85.0 & 17.0 \\
\hline & $\mathrm{F} 2$ & 33.9 & 1.53 & 1.79 & 16.7 & 90.0 & 4.85 \\
\hline & F3 & 18.8 & 1.52 & 1.68 & 5.8 & 110 & 1.90 \\
\hline & $\mathrm{F} 4$ & 5.97 & 1.34 & 1.45 & 3.5 & 140 & 0.45 \\
\hline
\end{tabular}

a The recovery was defined as a photometrically-determined weight of a sonicated and fractionated sample divided by the weight of the h sample. Values in parentheses are the sum total of the percent recovery of all fractionated samples.

b The volume of acetone that was added to separate each fraction divided by the volume of the s-sample solution.

c Because of high molecular weight, the elution volume was beyond the limit and the molecular distribution was undetermined.

${ }^{d}$ No GPC/LALLS measurement for the s sample.

e No GPC/LALLS measurement for the h sample, because of the high molecular weight. 
properties, the polydispersity of the system must be taken into account. The physical parameters involved in these properties depend on the method of measurement and are given by some averaged quantities, e.g., numberaverage or higher-order averages. ${ }^{30}$ In order to compare the observed data with a particular theoretical expression, use of a distribution function in an analytical form is often quite convenient. ${ }^{35}$ Among others, two analytical functions have been utilized in previous reports from our laboratory ${ }^{1,3,33,34}$; the Schultz$\mathrm{Zimm}^{36,37}$ (eq 5) and the logarithmic-normal, the Wesslau ${ }^{38}$ or the Lansing-Kraemer ${ }^{39}$ (eq 6) functions that are given below.

$$
f_{w}(M)=(-\ln p)^{k+1} M^{k} p^{M} / \Gamma(k+1)
$$

where $f_{w}(M)$ is the distribution function in terms of weight fraction, $M$ is the molecular weight, $\Gamma(k+1)$ is the gamma function, $M_{n}=$ $-k / \ln p, M_{w}=-(k+1) / \ln p, k=M_{n} /\left(M_{w}-M_{n}\right)$, and $p=\exp \left[-1 /\left(M_{w}-M_{n}\right)\right]$.

$$
f_{w}(M)=\frac{1}{\omega \sqrt{\pi}} \exp \left\{-\frac{1}{\omega^{2}}\left(\ln \frac{M}{M_{0}}\right)^{2}\right\} \cdot \frac{1}{M}
$$

where $M_{n}=M_{0} \exp \left(-\omega^{2} / 4\right), M_{w}=M_{0} \exp \left(\omega^{2} /\right.$ 4), $M_{0}=\left(M_{w} M_{n}\right)^{1 / 2}$. The Schulz-Zimm function has been utilized to express the molecular weight distribution of polymers synthesized by addition polymerization, while the Wesslau function has been shown to be useful for the products of the Ziegler-Natta polymerization.

Figure 3 shows the observed distribution profiles (solid lines) of the sonicated but unfractionated DNA sample (denoted as s) and a fractionated sample (denoted as F4), as compared with the theoretical Schulz-Zimm (dotted lines) and logarithmic-normal (dashed lines) distribution functions, each being calculated with observed $M_{w}$ and $M_{w} / M_{n}$ values. The observed distribution of the s sample is closer to the Schulz-Zimm function, whereas the F4 fraction can be fitted by neither function.

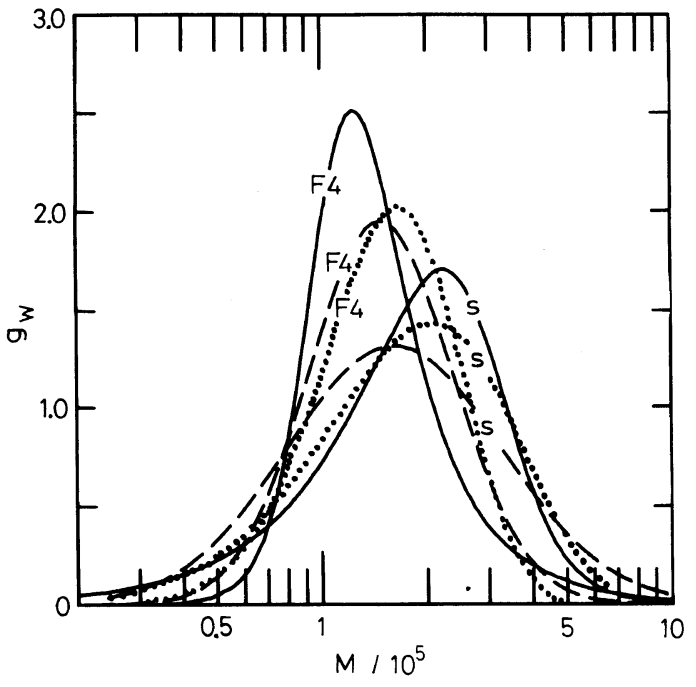

Figure 3. Comparison of the experimental molecular weight distribution profile (solid lines) of DNA with the theoretical logarithmic-normal (dashed lines) and SchulzZimm (dotted lines) distribution functions. (s), sonicated but unfractionated sample $\left(M_{w}=2.06 \times 10^{5}, M_{w} / M_{n}=\right.$ 1.64); (F4), sonicated and fractionated sample $\left(M_{w}=\right.$ $\left.1.65 \times 10^{5}, M_{w} / M_{n}=1.25\right)$.

Figure 4 shows the observed distribution profiles (solid lines) of the unsonicated starting $\operatorname{Poly}(\mathrm{A}) \cdot \operatorname{Poly}(\mathrm{U})$ sample (denoted as $\mathrm{h}$ ) and a fractionated one (denoted as F3). The h sample fits to neither of the theoretical functions, but the distribution of the F3 and the sonicated but unfractionated samples (the latter not shown here) can be reproduced quite well with the logarithmic-normal theoretical function. Thus, it is clear that the well-defined lower molecular weight $\operatorname{Poly}(\mathrm{A}) \cdot \operatorname{Poly}(\mathrm{U})$ sample with a narrow distribution can be prepared in a large quantity according to the present sonication-fractionation method.

Figure 5 shows the observed distribution (solid lines) of the unsonicated starting $\operatorname{Poly}(\mathrm{C}) \cdot \operatorname{Poly}\left(\mathrm{C}^{+}\right)$sample (denoted as $\mathrm{h}$ ) and a fractionated one (denoted as F3). The $h$ sample can be fitted approximately by the Schulz-Zimm function, but the fractionated F3 sample can be reproduced by neither of two theoretical distribution functions. This disagreement is probably due to the fact that the 


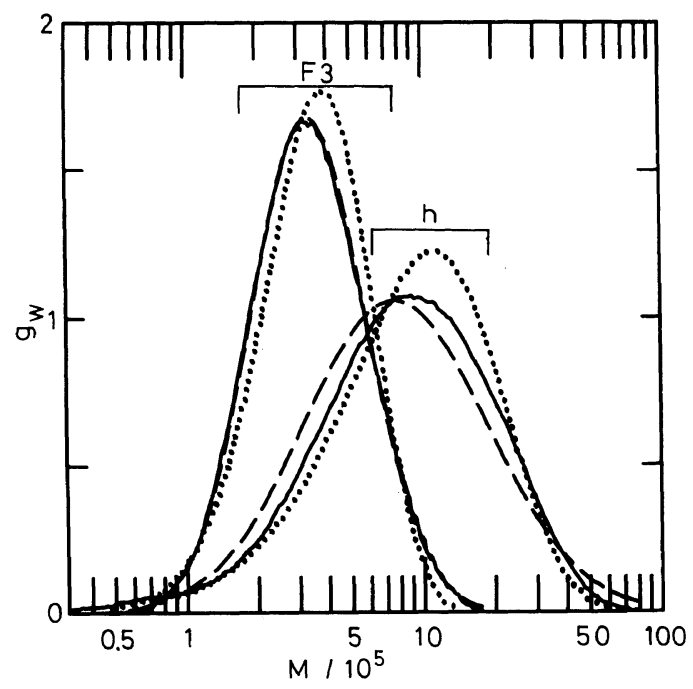

Figure 4. Comparison between the experimental molecular weight distribution profile of $\operatorname{Poly}(\mathrm{A}) \cdot \operatorname{Poly}(\mathrm{U})$ with theoretical distribution functions. (h), unsonicated sample $\left(M_{w}=1.13 \times 10^{6}, \quad M_{w} / M_{n}=2.17\right)$; (F3), sonicated and fractionated sample $\left(M_{w}=3.85 \times 10^{5}, \quad M_{w} / M_{n}=1.35\right)$. Other notations are all the same as in Figure 3.

higher and lower molecular weight portions still remain in the acetone-precipitate, in addition to the major component, just as in the case of DNA ( $c f$. Figure 3).

From the above comparisons between observed and theoretical distribution profiles for six polynucleotide duplexes (not all were shown in this section), the following general conclusion may be drawn on the molecular weight distribution. If the sonication-fractionation procedure is carried out properly, the molecular weight distribution of an acetoneprecipitated fraction is in practice represented by the logarithmic-normal distribution function, which has been utilized extensively in electrooptic studies. ${ }^{1,3,31-34}$ The present finding is an important confirmation of these previous studies. The data analysis may be practised with the theoretical distribution function $f_{w}(M)$ to evaluate the physical parameters involved in the hydrodynamic and other physico-chemical properties of polydisperse polymer systems.

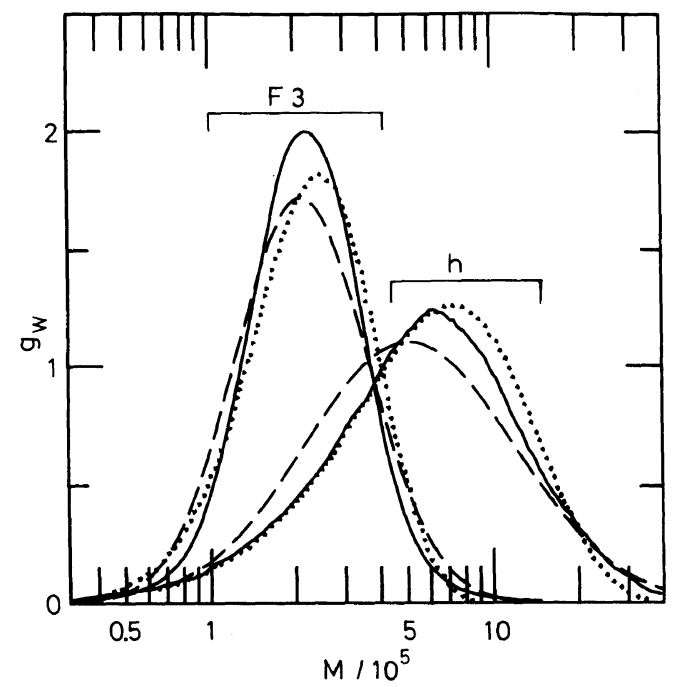

Figure 5. Comparison of the experimental molecular weight distribution profile of $\operatorname{Poly}(\mathrm{C}) \cdot \operatorname{Poly}\left(\mathrm{C}^{+}\right)$with theoretical distribution functions. (h), unsonicated sample $\left(M_{w}=7.26 \times 10^{5}, \quad M_{w} / M_{n}=2.04\right) ;(\mathrm{F} 3)$, sonicated and fractionated sample $\left(M_{w}=2.45 \times 10^{5}, \quad M_{w} / M_{n}=1.33\right)$. Other notations are all the same as in Figure 3.

\section{Intrinsic Viscosity of Unirradiated and Irra- diated Duplexes}

Figure 6 shows the variation of the reduced viscosity $\eta_{\text {sp }} / c^{\prime}$ with the mass concentration $c^{\prime}$ for two duplex samples in appropriate salt solutions. The reduced viscosities of the starting high molecular weight $\operatorname{Poly}(\mathrm{A}) \cdot$ Poly(U) in Figure 6a and Poly(I) Poly(C) in Figure $6 \mathrm{~b}$ (both denoted as $\mathrm{h}$ ) clearly depend on the shear rate. A double extrapolation to zero concentration and to zero shear rate must be carried out to evaluate the intrinsic viscosity $[\eta]$. The extrapolation to zero shear rate was achieved by plotting the bulb-height-dependent reduced viscosities of a given solution against $\left(c^{\prime}+k h^{2}\right)$, where $k$ is an arbitrarily chosen constant and $h$ is the average height of each bulb of a viscometer (the detail will be reported elsewhere). The reduced viscosities of the sonicated and fractionated preparations show a linear concentration-dependence without exception, the slope being smaller with lower $[\eta]$ values. The sonicated but unfractionated 

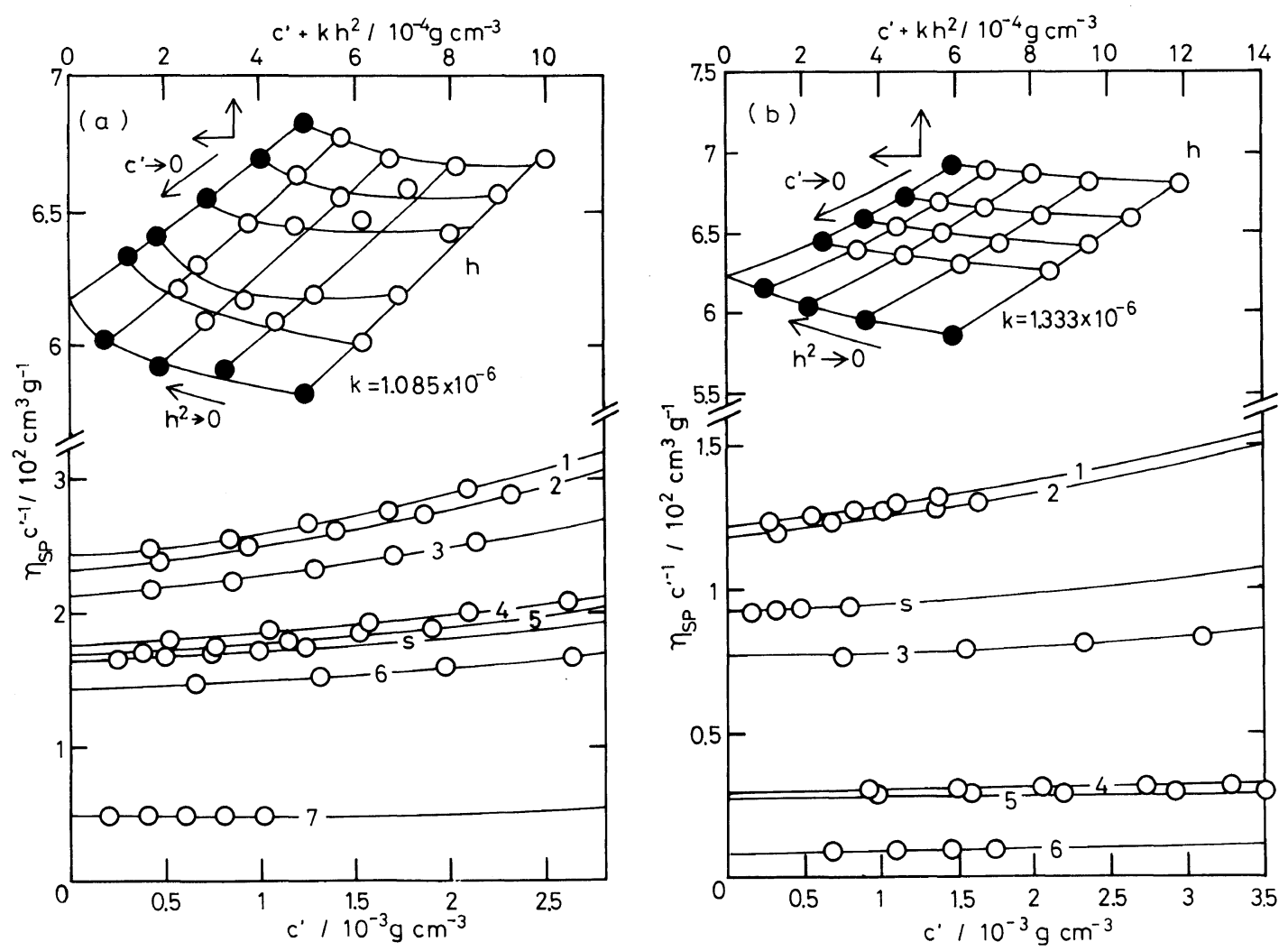

Figure 6. The reduced viscosity $\eta_{\mathrm{sp}} / c^{\prime}$ vs. the mass concentration $c^{\prime}$ at $25^{\circ} \mathrm{C}$. (a): $\operatorname{Poly}(\mathrm{A}) \cdot \operatorname{Poly}(\mathrm{U})$ in $0.1 \mathrm{M} \mathrm{NaCl}$. (b): Poly(I) $\cdot \operatorname{Poly}(\mathrm{C})$ in $0.2 \mathrm{M} \mathrm{NaCl}$. Numerals denote the fraction number of each sample (cf. Table I). $h$ is the average height between the middle of each bulb and the end of capillary spiral of a viscometer. $k$ is an arbitrary constant in $\mathrm{g} \mathrm{cm}^{-5}$ to spread observed values, being equal to $1.085 \times 10^{-6}$ for (a) and $1.333 \times 10^{-6}$ for (b). Filled circles are extrapolated points $\left(h^{2} \rightarrow 0\right.$ and $\left.c^{\prime} \rightarrow 0\right)$.

samples (denoted as s) yield $[\eta]$ values that are located appropriately between two fractionated preparations F5 and F6 for $\operatorname{Poly}(\mathrm{A})$. Poly(U) and between F2 and F3 for Poly(I). Poly(C). It should be noted that these results were also found for the other duplexes. The $[\eta]$ values are all given in Table I.

\section{Relationship between $[\eta]$ and $M_{w}$}

Figure 7 shows the double logarithmic plots of the relationship between the intrinsic viscosity and the weight-average molecular weight for six duplexes. A linear relationship was observed for every duplex, provided that the molecular weights are not higher than half a million. Since a sonicated but unfractionated sample (denoted as $\mathrm{s}$ with filled symbols) is associated with a wider molecular weight distribution, the contribution of the higher molecular weight portion to intrinsic viscosity may be proportionally larger, even if the weight-average molecular weight happens to be the same as that of the other sample. Thus, the points scattered from the solid lines in Figure 7 were not taken in evaluating the $[\eta]$ vs. $M_{w}$ relation, expressed as $[\eta]=K\left(M_{w}\right)^{\alpha}$, by the least-squares method. The constants $K$ and $\alpha$ are given in Table II, together with the range of molecular weights, in which the relationship holds.

The weight-average molecular weight can be estimated conveniently from the above re- 


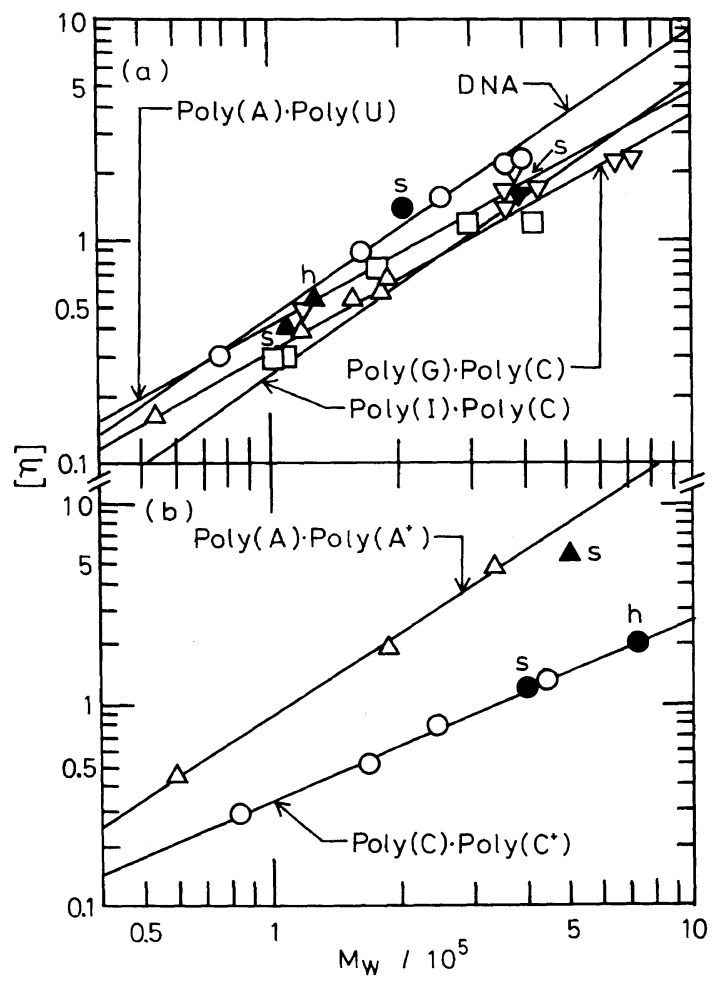

Figure 7. Relationship between $[\eta]$ and $M_{w}$ for six double-stranded helices on a double logarithmic scale. (a): measured points are $(\bigcirc)$ for DNA, $(\triangle)$ for $\operatorname{Poly}(G)$. Poly $(C),(\nabla)$ for Poly $(A) \cdot \operatorname{Poly}(U)$, and $(\square)$ for Poly(I) Poly $(C)$. (b): measured points are $(O)$ for $\operatorname{Poly}(C)$. $\operatorname{Poly}\left(\mathrm{C}^{+}\right)$and $(\triangle)$ for $\operatorname{Poly}(\mathrm{A}) \cdot \operatorname{Poly}\left(\mathrm{A}^{+}\right)$. Solid lines are drawn by the least-squares method. Filled symbols are either the unsonicated (h) or the sonicated but unfractionated (s) samples.

lationship not only for $\mathrm{DNA}^{10,13}$ but also for polyribonucleotide duplexes in a lower molecular weight range. The exponent $\alpha$ is in the range between 0.9 and 1.4 , indicating that the fractionated samples of all six duplexes are in a semiflexible conformation in $0.1-0.2 \mathrm{M} \mathrm{NaCl}$ solutions, ${ }^{30}$ although the degree of flexibility seems to vary with the polymer species. Whether this flexibility results from the pentose (the deoxy-vs. oxyribose) structure or from the regular or random sequence of hetero or homo base-pairs remains open to the future study. Since the antiparallel or parallel doublestrandedness of the helical structure appears
Table II. The parameters $K$ and $\alpha$ in the Mark-Houwink-Sakurada expression between intrinsic viscosity $[\eta]$ and weight-average molecular weight $M_{w}:[\eta]=K\left(M_{w}\right)^{\alpha}$

\begin{tabular}{|c|c|c|c|}
\hline \multirow{2}{*}{ Samples } & $K$ & & \multirow{2}{*}{$\begin{array}{c}\text { Applicable } \\
\text { range } \\
M_{w} / 10^{4}\end{array}$} \\
\hline & $10^{-6} \mathrm{~cm}^{3} \mathrm{~g}^{-1}$ & $\alpha$ & \\
\hline DNA & 14.1 & 1.30 & $7.6-39.9$ \\
\hline $\operatorname{Poly}(G) \cdot \operatorname{Poly}(C)$ & 138 & 1.07 & $5.3-19.1$ \\
\hline $\operatorname{Poly}(\mathrm{A}) \cdot \operatorname{Poly}(\mathrm{U})$ & 204 & 1.06 & $12.1-43.4$ \\
\hline $\operatorname{Poly}(\mathrm{I}) \cdot \operatorname{Poly}(\mathrm{C})$ & 6.16 & 1.32 & $5.7-42.3$ \\
\hline $\operatorname{Poly}(\mathrm{C}) \cdot \operatorname{Poly}\left(\mathrm{C}^{+}\right)$ & 933 & 0.91 & $8.4-44.7$ \\
\hline $\operatorname{Poly}(\mathrm{A}) \cdot \operatorname{Poly}\left(\mathrm{A}^{+}\right)$ & 13.8 & 1.36 & $6.0-33.9$ \\
\hline
\end{tabular}

unrelated to the $\alpha$ value, the size and the stacking of base-pairs may be responsible for the chain rigidity. The precise solution conformation of polynucleotides may be resolved with the sonicated fractions which can now be prepared in large quantities, as described in detail in this work.

\section{CONCLUSION}

This work showed that the ultrasonic scission can be performed successfully not only for high molecular weight calf thymus DNA but also for high molecular weight polyribonucleotide duplexes to reduce the molecular weight and polydispersity. The sonication-precipitational-fractionation technique developed in this series of work is simple but versatile. When this procedure is properly executed, a series of high-quality fractions can be prepared in large amounts from a single sonicated sample. Thus, the various physicochemical studies of nucleic acids in solutions can be advanced, where the molecular-weight dependence is often a critical factor.

The determination of the molecular weight and the molecular weight distribution of sonicated duplexes could be carried out conveniently by the GPC/LALLS method, if an appropriate gel material is selected for the GPC column. The present work revealed that 
the distribution profile of purified fractions can be fitted by the theoretical logarithmic-normal function. The relationship between intrinsic viscosity and weight-average molecular weight (the Mark-Houwink-Sakurada equation) was evaluated. The exponent $\alpha$ was found to be $1.4-0.9$ that represents the semiflexible chain.

Acknowledgments. The authors are indebted to Tosoh Co., Ltd. for generous permission for using a GPC/LALLS system, which was essential to the molecular weight determination. This work was in part supported by a Grant-in-Aid for Scientific Research ((A) No. 02405008 to KY) from the Ministry of Education, Science, and Culture.

\section{REFERENCES}

1. K. Matsuda and K. Yamaoka, Bull. Chem. Soc. Jpn., 55, 1727 (1982).

2. E. Charney and K. Yamaoka, Biochemistry, 21, 834 (1982).

3. K. Yamaoka and K. Fukudome, Bull. Chem. Soc. Jpn., 56, 60 (1983).

4. K. Yamaoka and K. Fukudome, J. Phys. Chem., 92, 4994 (1988).

5. K. Yamaoka and K. Fukudome, J. Phys. Chem., 94, 6896 (1990).

6. J. E. Godfrey, Biophys. Chem., 5, 285 (1976).

7. J. E. Godfrey and H. Eisenberg, Biophys. Chem., 5, 301 (1976).

8. K. Fukudome, K. Yamaoka, K. Nishikori, T. Takahashi, and O. Yamamoto, Polym. J., 18, 71 (1986).

9. K. Fukudome, K. Yamaoka, K. Nishikori, H. Tatehata, and O. Yamamoto, Polym. J., 18, 81 (1986).

10. K. Fukudome, K. Yamaoka, and H. Ochiai, Polym. J., 19, 1385 (1987).

11. K. Fukudome, K. Yamaoka, and M. Yamaguchi, Polym. J., 22, 937 (1990).

12. K. Yamaoka, K. Fukudome, N. Mukaiyama, H. Shirahama, and T. Suzawa, J. Colloid Interface Sci., 136, 519 (1990).
13. J. Eigner and P. Doty, J. Mol. Biol., 12, 549 (1965).

14. D. R. Davies and A. Rich, J. Am. Chem. Soc., 80, 1003 (1958).

15. H. Teitelbaum and S. W. Englander, J. Mol. Biol., 92, 79 (1975).

16. A. Rich and D. R. Davies, J. Am. Chem. Soc., 78, 3548 (1956).

17. C. L. Stevens and G. Felsenfeld, Biopolymers, 2, 293 (1964).

18. R. D. Blake, J. Massoulié, and J. R. Fresco, J. Mol. Biol., 30, 291 (1967).

19. S. Arnott, D. W. L. Hukins, S. D. Dover, W. Fuller, and A. R. Hodgson, J. Mol. Biol., 81, 107 (1973).

20. G. D. Fasman, C. Lindblow, and L. Grossman, Biochemistry, 3, 1015 (1964).

21. E. O. Akinrimisi, C. Sander, and P. O. P. Ts'o, Biochemistry, 2, 340 (1963).

22. R. Langridge and A. Rich, Nature, 198, 725 (1963).

23. K. A. Hartman, Jr. and A. Rich, J. Am. Chem. Soc., 87, 2033 (1965).

24. A. J. Adler, L. Grossman, and G. D. Fasman, Biochemistry, 8, 3846 (1969).

25. J. R. Fresco and P. Doty, J. Am. Chem. Soc., 79, 3928 (1957).

26. A. Rich, D. R. Davies, F. H. C. Crick, and J. D. Watson, J. Mol. Biol., 3, 71 (1961).

27. F. Pochon and A. M. Michelson, Proc. Natl. Acad. Sci. U.S.A., 53, 1425 (1965).

28. F. Pochon and A. M. Michelson, Proc. Natl. Acad. Sci. U.S.A., 57, 1441 (1965).

29. R. O. Day, N. C. Seeman, J. M. Rosenberg, and A. Rich, Proc. Natl. Acad. Sci. U.S.A., 70, 849 (1973).

30. C. Tanford, "Physical Chemistry of Macromolecules," John Wiley \& Sons, New York, N.Y., 1961, Chapters 3 and 6.

31. K. Ueda, M. Nomura, and K. Yamaoka, Biopolymers, 22, 2077 (1983).

32. K. Ueda, Bull. Chem. Soc. Jpn., 57, 2703 (1984).

33. K. Yamaoka and K. Ueda, J. Phys. Chem., 86, 406 (1982).

34. K. Matsuda and K. Yamaoka, Bull. Chem. Soc. Jpn., 55, 69 (1982).

35. P. J. Flory, "Principles of Polymer Chemistry," Cornell Univ. Press, Ithaca, N.Y., 1953, Chapter VIII.

36. G. V. Schulz, Z. Phys. Chem., B43, 25 (1939).

37. B. H. Zimm, J. Chem. Phys., 16, 1099 (1948).

38. H. Wesslau, Makromol. Chem., 20, 111 (1956).

39. W. D. Lansing and E. O. Kraemer, J. Am. Chem. Soc., 57, 1369 (1935). 